\title{
Measurement of backscatter to the monitor chamber of medical accelerators using target charge
}

\author{
Kwok L. Lam, Moorthy S. Muthuswamy, and Randall K. Ten Haken ${ }^{\mathrm{a}}$ \\ Department of Radiation Oncology, University of Michigan, Ann Arbor, Michigan 48109-0010
}

(Received 2 May 1997; accepted for publication 12 December 1997)

\begin{abstract}
A simple noninvasive method is described for determining the backscatter to a monitor chamber of a medical accelerator based on the measurement of charge deposited in the target. This method is compared quantitatively to the more elaborate telescopic method for photon beams of $6 \mathrm{MV}$ and 15 MV on linear accelerators having mica and Kapton monitor chambers. The new target charge method gives results consistent with the telescopic method to within 0.3\%. (C) 1998 American Association of Physicists in Medicine. [S0094-2405(98)00603-8]
\end{abstract}

Key words: backscatter, monitor chamber, target charge, telescope

\section{INTRODUCTION}

Changes in the amount of radiation backscattered from collimators to the monitor chamber can affect the energy fluence per monitor unit of a photon beam. ${ }^{1-6}$ Also, it has been found to be a contributing factor in collimator exchange effect. ${ }^{1}$ The contribution of backscattered radiation to the charge collected by the monitor chamber depends on the setting of the collimators. Thus the amount of charge collected by the monitor chamber for a given number of bremsstrahlung photons generated at the target changes as the collimator setting is varied from minimum to maximum. The percentage change ranges from less than $0.5 \%$ to about $10 \% .^{1-9}$ The origin of this wide range of dependence can be traced to differences in the design of the collimator ${ }^{4}$ and the monitor chamber. ${ }^{5}$ The dosimetric effect of backscattered radiation to the monitor chamber can be accounted for using monitor backscatter factors. ${ }^{1,2}$ These factors specify the number of bremsstrahlung photons generated at the target per monitor unit at a given collimator setting normalized to that at a reference collimator setting $[M S(w)$ in Patterson and Shragge ${ }^{1}$ and $S_{b}\left(r_{x}, r_{y}\right)$ in Lam et al. $\left.{ }^{2}\right]$. Since the monitor backscatter factor $\left(S_{b}\right)$ due to backscatter to the monitor chamber and the head scatter factor due to scattered radiation in the treatment head depend differently on the collimator setting, ${ }^{2}$ it is important to quantify backscatter to the monitor chamber.

Photoactivation, ${ }^{1}$ beam on time with beam current feedback disabled, ${ }^{7}$ number of beam pulses, ${ }^{6-8}$ addition of an attenuator between the collimator and the monitor chamber, ${ }^{3}$ target charge, ${ }^{9}$ and forward photon fluence from the target with telescopic collimation ${ }^{2,4-6}$ have been used to quantify backscatter to the monitor chamber. The methods differ in how the number of bremsstrahlung photons generated at the target for a given number of monitor units is measured. Photoactivation and addition of an attenuator require invasive introduction of test components into the treatment head. Beam on time and number of beam pulses depend on the accelerator to be stable. It has been found that fluctuations in pulse counts could be more than $10 \%$ on some machines. ${ }^{6}$ The target charge method was suggested by Patterson and
Shragge $^{1}$ and was used by Watts and Ibbott. ${ }^{9}$ In their method, invasive change to the circuitry of the linear accelerator was necessary. The target current circuit of the accelerator was interrupted, the target current was reduced by three orders of magnitude by a shunt circuit, and the target charge was then measured with an electrometer. The telescopic method introduced by Kubo ${ }^{4}$ involves the handling of heavy custom made collimators.

Here, the primary purpose was to improve the measurement of the target charge by design and implementation of a simple circuit totally external to the accelerator electronics so that no modification to the accelerator is necessary. We have also improved the technique for measuring backscatter with the telescopic method so that components of the setup are physically easy to handle and scatter from the collimation system and the room can be estimated more accurately. The consistency of the monitor backscatter factors measured with these two methods on various linear accelerators manufactured by Varian with different photon energies will be demonstrated.

\section{MATERIALS AND METHODS}

The measurements were made on a Clinac 6/100, a Clinac 1800 , and a Clinac 2100C/D. The x-ray energy on the Clinac 6/100 was $6 \mathrm{MV}$. The Clinac 1800 and Clinac 2100C/D were dual energy machines with $6 \mathrm{MV}$ and $15 \mathrm{MV}$ x-ray beams. The Clinac 6/100 had a mica monitor chamber and Kapton chambers were installed in the Clinac 1800 and the Clinac $2100 \mathrm{C} / \mathrm{D}$. The steel exit window of the mica chamber attenuated backscattered electrons from the collimators and provided a source of electrons while the Kapton exit window of a Kapton chamber transmitted backscattered electrons which contributed to the ionizations collected in the chamber. ${ }^{5}$ The $S_{b}$ 's were measured with both the target charge method and the telescopic method on all five x-ray beams, as described below.

In Varian Clinac linear accelerators, the target was insulated from electrical ground and connected through a coaxial cable to a $50 \mathrm{ohm}$ load (Fig. 1). This signal was available at a connector labeled "Target I' ("Beam I' on older models 


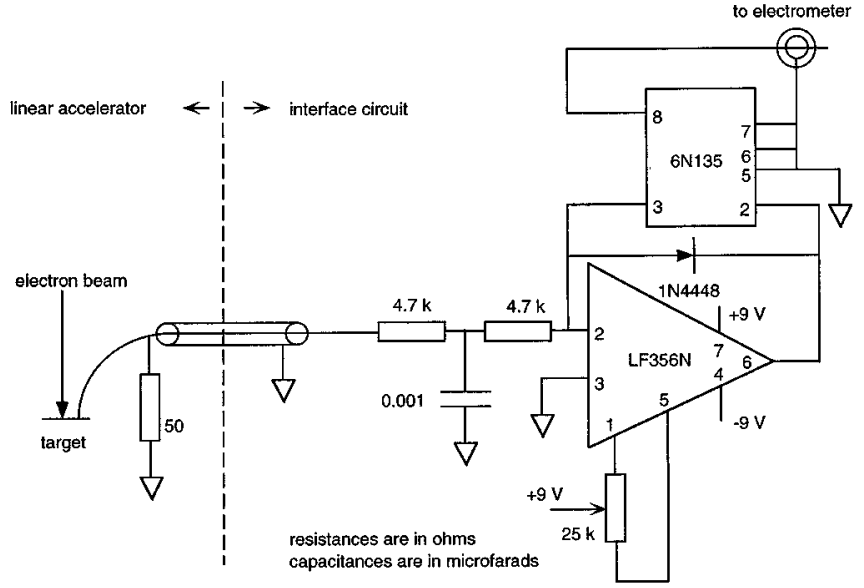

FIG. 1. Interface circuit to convert the voltage pulse at the target current output from the accelerator to a signal that the electrometer can integrate. The LF356N is a FET input operational amplifier. The 6 N135 is an optocoupler with a phototransistor output and the collector-base junction of the phototransistor is used as a photodiode to improve frequency response. The $1 \mathrm{~N} 4448$ is a silicon signal diode.

of Clinac). The current collected by the target generated a voltage on the order of $1 \mathrm{~V}$ across the $50 \mathrm{ohm}$ load. We assumed that a potential change of about one volt on the target would not significantly affect the current deposited from an electron beam of several $\mathrm{MeV}$ so that the voltage across the $50 \mathrm{ohm}$ load would be proportional to the electron current generating bremsstrahlung photons in the target. We used a circuit as shown in Fig. 1 powered by two $9 \mathrm{~V}$ batteries to measure the target charge. The operational amplifier LF356N converted the voltage to a current through the optoisolator $6 \mathrm{~N} 135$. The output of $6 \mathrm{~N} 135$ was then integrated by a Keithley 616 electrometer. The $25 \mathrm{k} \Omega$ potentiometer in the circuit was adjusted so that the output of the operational amplifier (pin 6) was at $-30 \mathrm{mV}$ when the input was disconnected from the accelerator. This ensured that there was no current generated at the output to the electrometer when the beam was off.

The target charges for 100 monitor units (MU) were determined for upper jaw settings varied from $0.5 \mathrm{~cm}$ to $40 \mathrm{~cm}$ with the lower jaws held fixed at $40 \mathrm{~cm}$ as well as lower jaw settings varied from $0.5 \mathrm{~cm}$ to $40 \mathrm{~cm}$ with the upper jaws fixed at $40 \mathrm{~cm}$. The target charge at $40 \mathrm{~cm}$ by $40 \mathrm{~cm}$ was measured repeatedly during the course of the measurement to monitor the stability of the machine. The target charges were then normalized to the average reading at $40 \mathrm{~cm}$ by 40 cm field size setting to obtain $S_{b}$ 's.

For the telescopic method, the experimental setup consists of a detector housing and a lead block collimator with the gantry oriented so that the x-ray beam was directed vertically towards the floor as shown in Fig. 2. The detector housing was on the floor at $230 \mathrm{~cm}$ from the target. The lead block collimator was on the accessory mount. The bottoms of the lead block collimators were $65 \mathrm{~cm}$ and $68.5 \mathrm{~cm}$ from the target for the Clinac 6/100 and the Clinac 2100C/D, respectively.

The detector housing consisted of a stack of two $7.6 \mathrm{~cm}$
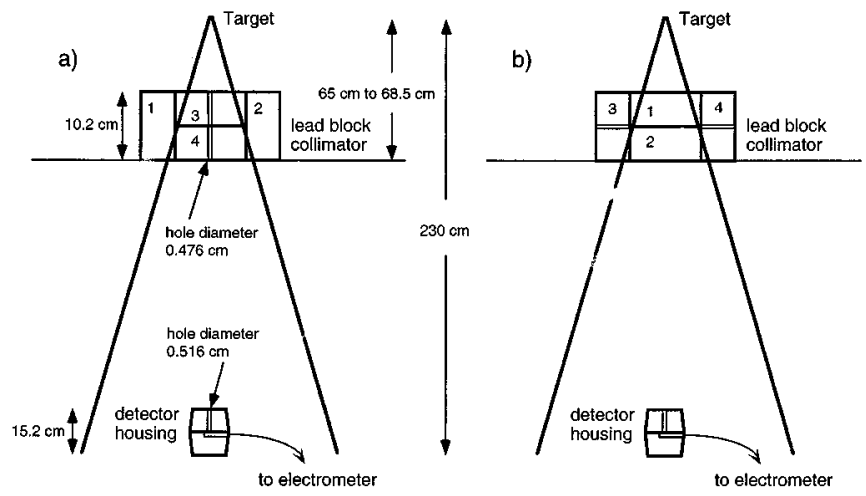

FIG. 2. Experimental setup for the telescopic method. (a) The setup for the measurement of collimated and scattered radiation. The distance between the target and the bottom of the lead block collimator was $65 \mathrm{~cm}$ for Clinac 6/100 and Clinac 1800 and $68.5 \mathrm{~cm}$ for Clinac $2100 \mathrm{C} / \mathrm{D}$. (b) The setup for the measurement of scattered radiation. The lead blocks numbered 1 to 4 on the accessory mount were rearranged from (a).

thick blocks of Cerrobend $10.5 \mathrm{~cm}$ wide by $10.5 \mathrm{~cm}$ long. An IC-10 chamber (Wellhofer Dosimetrie) was in the middle of the detector housing as shown in Fig. 2. The chamber with its $1 \mathrm{~mm}$ thick cap was in a horizontal channel on the top surface of the lower block of the housing. A $0.516 \mathrm{~cm}$ diameter vertical hole in the upper block of the housing collimated the $\mathrm{x}$ rays before they were detected by the chamber.

The detection solid angle was determined by the lead block collimator consisting of a stack of two lead blocks placed on the accessory mount with $0.476 \mathrm{~cm}$ diameter holes along the central axis of the x-ray beam [blocks 3 and 4 in Fig. 2(a)]. The lead blocks were $5.1 \mathrm{~cm}$ thick, $10.2 \mathrm{~cm}$ wide, and $20.3 \mathrm{~cm}$ long. One similar lead block was placed on each side of the stack with the $10.2 \mathrm{~cm}$ side vertical [blocks 1 and 2 in Fig. 2(a)]. The four lead blocks together formed a $20.3 \mathrm{~cm} \times 20.3 \mathrm{~cm} \times 10.2 \mathrm{~cm}$ structure. The holes in the lead blocks, the hole in the Cerrobend housing and the chamber were aligned by the cross hair of the light field, and the alignment was further optimized by maximizing the charge collected by the chamber.

Charges collected by the chamber for $500 \mathrm{MU}$ were recorded with the upper jaws varied from $3 \mathrm{~cm}$ to $40 \mathrm{~cm}$ settings when the lower jaws were kept fixed at $40 \mathrm{~cm}$ as well as with the lower jaws varied from $3 \mathrm{~cm}$ to $40 \mathrm{~cm}$ settings when the upper jaws were kept fixed at $40 \mathrm{~cm}$. The collimator setting at $40 \mathrm{~cm}$ by $40 \mathrm{~cm}$ were measured repeatedly during the course of the measurement. To estimate the amount of scattered radiation, the lead blocks with holes were switched in position with the lead blocks on the sides so that there was no hole along the central axis [Fig. 2(b)]. The measurements were then repeated for the same jaw settings. The charges from scattered radiation were subtracted from that collected with the telescope and the results were normalized to the average value of the $40 \mathrm{~cm} \times 40 \mathrm{~cm}$ field size setting.

We limited the smallest field size to $3 \mathrm{~cm}$ and tracked the trend of the data between $3 \mathrm{~cm}$ and $5 \mathrm{~cm}$ field sizes for the telescopic method to ensure that the upper jaws did not obscure the area of the target that was visible from the detector through the lead block collimator. For the target charge 
method, all these precautions are unnecessary because data can be obtained at any field size (including zero field size). We decided to replace the 3,4 , and $5 \mathrm{~cm}$ measurements by a measurement at $0.5 \mathrm{~cm}$, the minimum jaw setting for the Clinac 2100C/D.

\section{RESULTS}

The $S_{b}$ 's for the five beams are shown in Fig. 3. Results from the telescopic method and the target charge method are within $0.3 \%$ for all the beams measured. The dependencies of $S_{b}$ on collimator setting are fit to a linear function by the least squares method. The slope of the fit to the data, which is the change in $S_{b}$ per cm of change in collimator setting, are shown in Table I. The uncertainties shown in the telescope and the target charge are standard errors of the slopes estimated from linear least squares fitting of the data. The last column is the difference between the two methods and the uncertainties shown are the sum in quadrature of the standard errors of the two methods. The results for the two methods shown in Table I are highly correlated with a Pearson Correlation of 0.99 . A paired $t$-test results in insignificant $(P=0.47$, two tail $)$ difference in the mean of the results in Table I for the two methods.

\section{DISCUSSION}

The dependence of $S_{b}$ on jaw opening shown in Fig. 3 can be approximated well by a linear dependence for all machines studied. This is consistent with the parametrization proposed by Lam et al. ${ }^{2}$ and results reported by Kubo, ${ }^{4} \mathrm{Yu}$ et $a{ }^{6}{ }^{6}$ (for jaw settings $>5 \mathrm{~cm}$ ), and Lam et al. ${ }^{2}$ Thus the data were analyzed by the linear least squares method. The $S_{b}$ of Clinac $6 / 100$ measured by both the target charge method and the telescopic method show little dependence on jaw settings, similar to the results reported ${ }^{6}$ with the target pulse counting method and the telescopic method. This is due to backscattered electrons being stopped by the steel exit window of its mica monitor chamber. ${ }^{5}$ The stronger dependence on jaw settings for the other two accelerators is due to the transmission of backscattered electrons through the thin windows of the Kapton monitor chambers. ${ }^{5}$ The high energy beams on both the Clinac 1800 and Clinac 2100C/D showed a larger change in $S_{b}$ than the corresponding low energy beams when collimator setting was varied. This is also supported by previous reports. ${ }^{5,6}$

The mean of the differences in the last column of Table I is $0.1 \times 10^{-4} \mathrm{~cm}^{-1}$ with a standard deviation of 0.4 $\times 10^{-4} \mathrm{~cm}^{-1}$, indicating that there was no systematic difference between the results of the two methods. The standard deviation compares well with standard error estimates shown in the last column of Table I. Together with the fact that the results of the two methods were highly correlated and there was no significant difference in the mean from the paired $t$-test, we consider the results measured by the target charge method and the telescopic method to be consistent with each other.

For the telescopic method, the collimating hole in the detector housing in front of the detector determines the field of view for scattered radiation. The field of view usually covers the additional collimator (the four lead blocks in our setup) that constitutes the telescope. For example, in the measurement of $S_{b}$ for the $15 \mathrm{MV}$ beam on the Clinac 1800, the chamber reading for the scattered radiation was $1.3 \%$ of that from the telescope when the jaws were fully open and it was $0.6 \%$ when one of the jaws were set to $3 \mathrm{~cm}$. Since the small change in signal for different jaw settings is measured, the change in scattered radiation can be significant.

When Kubo introduced the telescopic method, ${ }^{4}$ he measured the scattered radiation with a separate chamber positioned next to the signal chamber away from the slit. $\mathrm{Yu}$ et $a l .{ }^{6}$ measured the scattered radiation with no hole in any of the lead blocks. Lam et l. $^{2}$ measured the scattered radiation by filling up the hole in the collimator mounted on the accessory tray and leaving the hole in the detector housing open. The radiations detected can be grouped into three catagories: (1) direct $\mathrm{x}$ rays from the target passing through the holes in the collimators; (2) $\mathrm{x}$ rays scattered from the direct $\mathrm{x}$ rays; and (3) scattered $\mathrm{x}$ rays from $\mathrm{x}$ rays that did not pass through the collimating holes of the telescope. Scattered $x$ rays in category 2 are proportional to the direct $\mathrm{x}$ rays while the scattered $x$ rays in category 3 have a different field size dependence compared to direct $x$ rays. The goal of all these three different methods is to estimate the scattered $\mathrm{x}$ rays which had a different field size dependence (category $3 \mathrm{x}$ rays) so that they can be subtracted from the signal. In our measurements, scattered $x$ rays from the room and scattered $\mathrm{x}$ rays from the lead block collimator were in category 3 while the scattered $\mathrm{x}$ rays from direct $\mathrm{x}$ rays scattered off the detector housing were in category 2. Blocking the holes in the lead blocks on the accessory mount attenuated as much direct radiation as blocking the holes in the detector housing. However, blocking the holes in the detector housing will attenuate both the direct $\mathrm{x}$ rays and scattered radiations from the lead blocks collimator. We thus followed the method used in reference 2 for the estimation of scattered radiation.

We have used four standard lead blocks that are commercially available for shielding purposes with some minor drilling that can be done in a typical field shaping block fabrication room to make the telescope. The arrangement is designed to make the procedure simple and not physically demanding to set up. The measurement of scattered radiation is done by rearranging the lead blocks so that only four lead blocks need to be handled. However, we have found that typical plastic trays for field shaping blocks need to be reinforced to support the weight of the four lead blocks.

During our measurements of target charge, we simultaneously monitored the target pulses at the "Target I" connector of the linear accelerator with an oscilloscope equipped with pulse counting capability (Tektronics 2247A). On the $15 \mathrm{MV}$ beam of the Clinac $2100 \mathrm{C} / \mathrm{D}$, we observed fluctuations in pulse counts of $8.5 \%$ at a fixed collimator setting, similar to what was reported by $\mathrm{Yu}$ et al. ${ }^{6}$ on some of their Clinac 600Cs. The simultaneously measured target charge fluctuated by less than $0.2 \%$. This indicates that the target charge method is immune to the fluctuations that affect target pulse count. 
a) Clinac $2100 \mathrm{CD} 15 \mathrm{MV}$

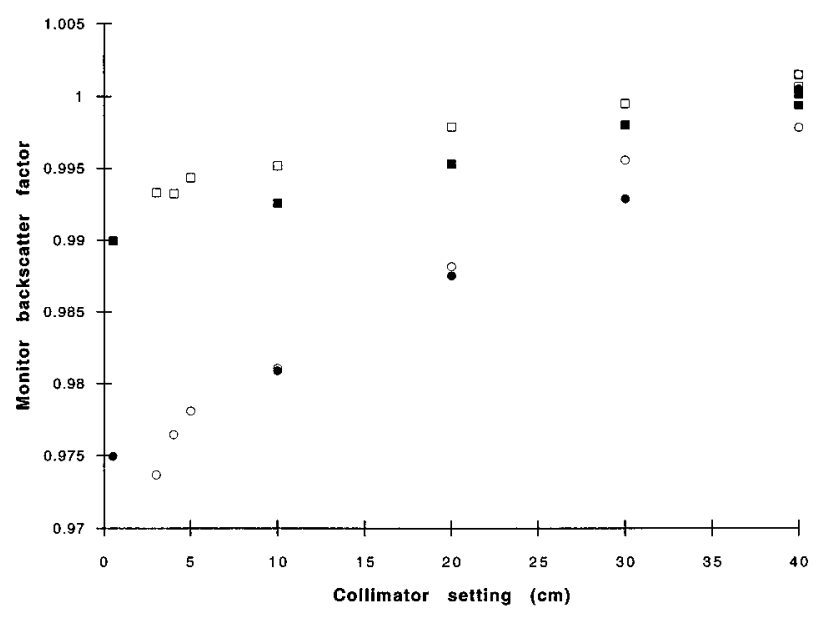

b) Clinac $2100 \mathrm{CD} 6 \mathrm{MV}$

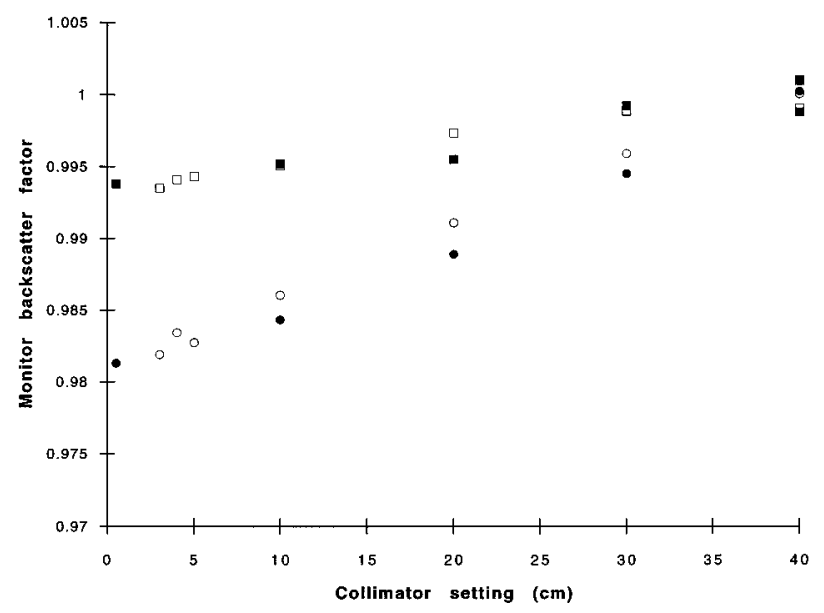

c) Clinac $180015 \mathrm{MV}$

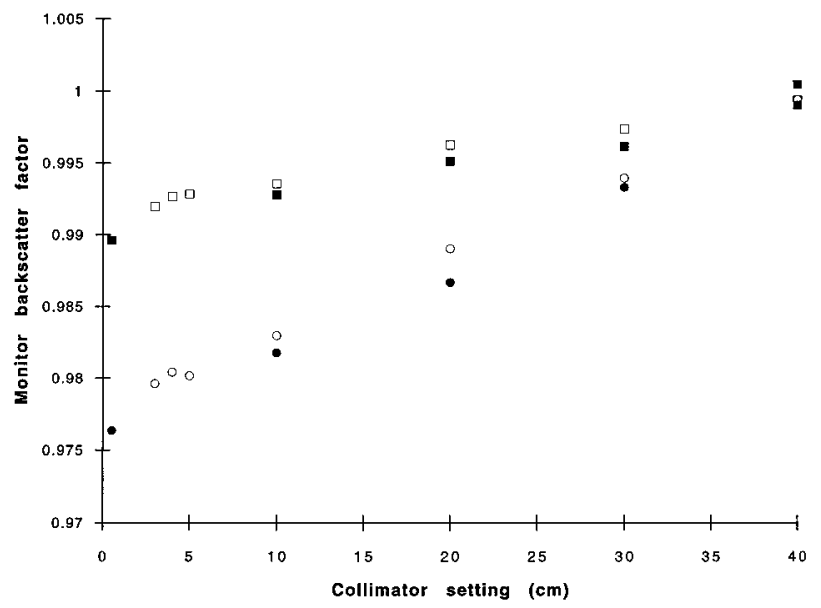

The "Target I" signal is usually provided as a diagnostic tool for the service person. It is not usually tested in the acceptance test procedure of the linear accelerator. Also, its condition may not be maintained during the life of the treatment machine. For example, the electrical connections at the connectors and through the coaxial cable may degrade; or the electrical grounding may change. Although the target charge method is found to be immune to fluctuations, from the point d) Clinac 1800 6MV

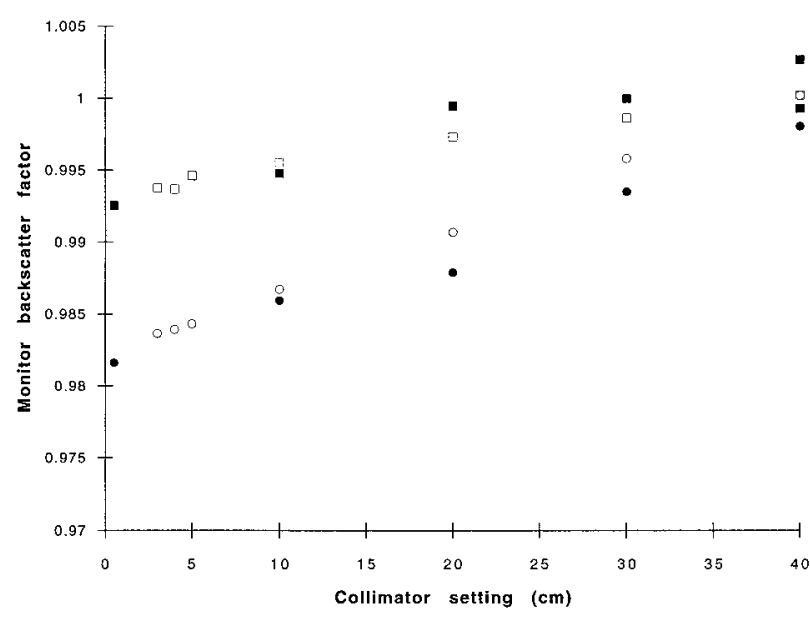

e) Clinac 6/100 6MV

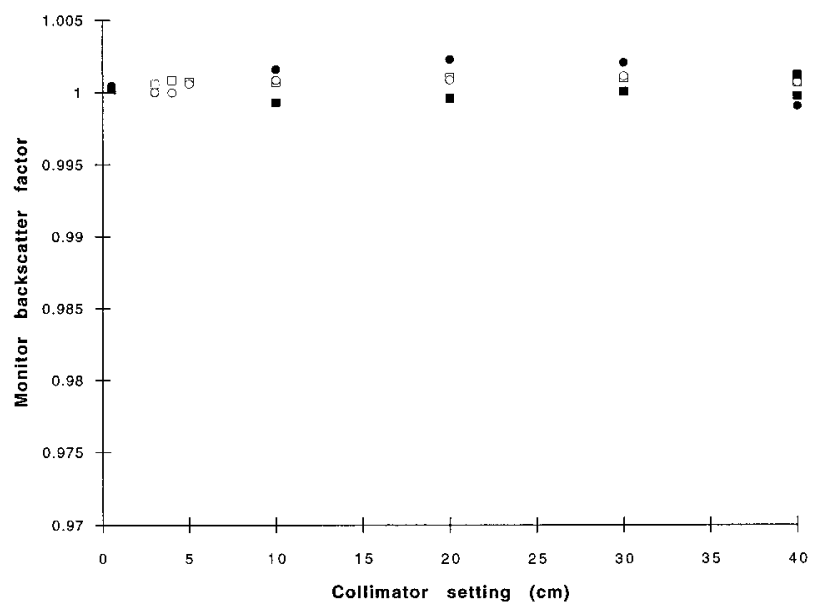

FIG. 3. Monitor backscatter factor for the three accelerators and the two photon energies. (a) Clinac $2100 \mathrm{C} / \mathrm{D}, 15 \mathrm{MV}$. (b) Clinac $2100 \mathrm{C} / \mathrm{D}, 6 \mathrm{MV}$. (c) Clinac 1800, 15 MV. (d) Clinac 1800, 6 MV. (e) Clinac 6/100, 6 MV. Open symbol data points were measured with the telescopic method and solid symbol data points were measured with the target charge method. Circular symbol data points were the dependence of monitor backscatter factor on upper jaw settings when the lower jaws were set at $40 \mathrm{~cm}$. Square symbol data points were the dependence of monitor backscatter factor on lower jaw settings when the upper jaws were set at $40 \mathrm{~cm}$.

of view of quality assurance, it is still recommended that the target current signal be checked by a qualified person to ensure that the signal exists, the amplitude is normal and the pulse shape is acceptable before it is used for $S_{b}$ measurements.

The target charge method is simple to setup and it can be implemented without invasive modification to the linear accelerator. The measurements can be obtained for any jaw 
TABLE I. Change in monitor backscatter factor $\left(S_{b}\right)$ per unit change in collimator setting from the slope of the data shown in Fig. 3. The uncertainties shown are standard errors.

\begin{tabular}{|c|c|c|c|}
\hline & $\begin{array}{l}\text { Telescope } \\
\left(10^{-4} / \mathrm{cm}\right)\end{array}$ & $\begin{array}{l}\text { Target charge } \\
\left(10^{-4} / \mathrm{cm}\right)\end{array}$ & $\begin{array}{l}\text { Difference } \\
\left(10^{-4} / \mathrm{cm}\right)\end{array}$ \\
\hline $\begin{array}{l}\text { Clinac } 1800 \\
15 \mathrm{MV} \\
\text { Upper Jaws }\end{array}$ & $5.5 \pm 0.2$ & $6.0 \pm 0.2$ & $-0.5 \pm 0.3$ \\
\hline $\begin{array}{l}\text { Clinac } 2100 \mathrm{C} / \mathrm{D} \\
15 \mathrm{MV} \\
\text { Upper Jaws }\end{array}$ & $6.7 \pm 0.3$ & $6.3 \pm 0.1$ & $0.4 \pm 0.3$ \\
\hline $\begin{array}{l}\text { Clinac } 1800 \\
15 \mathrm{MV} \\
\text { Lower Jaws }\end{array}$ & $2.1 \pm 0.2$ & $2.5 \pm 0.2$ & $-0.4 \pm 0.3$ \\
\hline $\begin{array}{l}\text { Clinac } 2100 \mathrm{C} / \mathrm{D} \\
15 \mathrm{MV} \\
\text { Lower Jaws }\end{array}$ & $1.8 \pm 0.3$ & $2.5 \pm 0.1$ & $-0.7 \pm 0.3$ \\
\hline $\begin{array}{l}\text { Clinac } 1800 \\
6 \mathrm{MV} \\
\text { Upper Jaws }\end{array}$ & $4.5 \pm 0.1$ & $4.7 \pm 0.5$ & $-0.2 \pm 0.5$ \\
\hline $\begin{array}{l}\text { Clinac } 2100 \mathrm{C} / \mathrm{D} \\
6 \mathrm{MV} \\
\text { Upper Jaws }\end{array}$ & $4.8 \pm 0.2$ & $4.9 \pm 0.3$ & $-0.1 \pm 0.4$ \\
\hline $\begin{array}{l}\text { Clinac } 1800 \\
6 \text { MV } \\
\text { Lower Jaws }\end{array}$ & $1.6 \pm 0.1$ & $1.8 \pm 0.5$ & $-0.2 \pm 0.5$ \\
\hline $\begin{array}{l}\text { Clinac } 2100 \mathrm{C} / \mathrm{D} \\
6 \mathrm{MV} \\
\text { Lower Jaws }\end{array}$ & $1.7 \pm 0.1$ & $1.7 \pm 0.3$ & $0.0 \pm 0.3$ \\
\hline $\begin{array}{l}\text { Clinac } 6 / 100 \\
6 \text { MV } \\
\text { Upper Jaws }\end{array}$ & $0.2 \pm 0.1$ & $-0.4 \pm 0.3$ & $0.6 \pm 0.3$ \\
\hline $\begin{array}{l}\text { Clinac } 6 / 100 \\
6 \mathrm{MV} \\
\text { Lower Jaws }\end{array}$ & $0.04 \pm 0.05$ & $-0.1 \pm 0.2$ & $0.1 \pm 0.2$ \\
\hline
\end{tabular}

setting available to the machine including zero jaw setting. The $S_{b}$ measured on different machines with similar head design were within $0.5 \%$. It may be possible to establish a standard $S_{b}$ for each design of linear accelerator treatment head so that only spot checks with target charge measurements would be necessary.

\section{CONCLUSIONS}

We have demonstrated that monitor backscatter factor can be measured conveniently without invasive modification to the linear accelerator with the target charge method. The target charge method and the telescope method with scattered radiation correction give consistent results in the measurement of monitor backscatter factor.

\section{ACKNOWLEDGMENT}

This work was supported in part by NIH Grant No. P01CA59827.

${ }^{a)}$ Electronic-mail: rth@umich.edu

${ }^{1}$ M. S. Patterson and P. C. Shragge, "Characteristics of an 18 MV photon beam from a Therac 20 Medical Linear Accelerator,' Med. Phys. 8, 312-318 (1981).

${ }^{2}$ K. L. Lam, M. S. Muthuswamy, and R. K. Ten Haken, "Flattening-filterbased empirical methods to parametrize the head scatter factor," Med. Phys. 23, 343-352 (1996).

${ }^{3}$ G. Luxton and M. A. Astrahan, "Output factor constituents of highenergy photon beam,' Med. Phys. 15, 88-91 (1988).

${ }^{4} \mathrm{H}$. Kubo, "Telescopic measurements of backscattered radiation from secondary collimator jaws to a beam monitor chamber using a pair of slits," Med. Phys. 16, 295-298 (1989).

${ }^{5}$ C. Duzenli, B. McClean, and C. Field, "Backscatter into the beam monitor chamber: Implications for dosimetry of asymmetric collimators,", Med. Phys. 20, 363-367 (1993).

${ }^{6}$ M. K. Yu, R. S. Sloboda, and F. Mansour, "Measurement of photon beam backscatter from collimators to the beam monitor chamber using target-current-pulse-counting and telescope techniques," Phys. Med. Biol. 41, 1107-1117 (1996).

${ }^{7} \mathrm{P}-\mathrm{H}$. Huang, J. Chu, and B. E. Bjärngard, "The effect of collimator backscatter radiation on photon output of linear accelerators,' Med. Phys. 14, 268-269 (1987).

${ }^{8}$ M. B. Sharpe, D. A. Jaffray, J. J. Battista, and P. Munro, “Extrafocal radiation: A unified approach to the prediction of beam penumbra and output factors for megavoltage x-ray beams,' Med. Phys. 22, 2065-2074 (1995).

${ }^{9}$ D. L. Watts and G. S. Ibbott, "Measurement of beam current and evaluation of scatter production in an 18-MeV accelerator,' Med. Phys. 14, 662-664 (1987). 